\title{
Formalgenese der Harnsteine
}

\section{Symposium vom 4. bis 5. Januar 1958 in Köln}

Teilnehmer:

KoUoidchemie: Prof. Dr. H. Thiele, Lehrfach der KoUoidchemie, Universität Kiel. Prof. Dr. Erbring, Biolog. Institut Madaus, Köln.

Physiol. Chemie: Dr. H. J. Dulce, Physiol.-Chem. Institut der Freien Universität Berlin. Dr. G. Hermann, Urologische Universitätsklmik, Homburg/Saar.

Biologie: Dr. Dr. Fr. E. Koch, Biolog. Institut Madaus, Köln.

Dr. H. Haase, Biolog. Institut Madaus, Köln.

Pathologie: Prof. Dr. M. Staemmler, Pathol. Institut der Stadt Aachen.

Dr. H. Uebel, Biolog. Institut Madaus, Köln.

Mineralogie: Prof. Dr. H. v. Philipsborn, Mineralog. Institut der Universität Bonn.

Doz. Dr. A. Preisinger, Mineralog. Institut der Universität Wien.

Innere Medizin: Prof. Dr. G. Orzechowski, Köln.

Dr. S. Wernitz, Städt. Krankenanstalten, Wuppertal-Barmen.

Urologie: $\quad$ Prof. Dr. C. E. Alken, Urol. Universitätsklinik, Homburg/Saar.

Prof. Dr. K. Boshamer, Städt. Krankenanstalten, Wuppertal-Barmen.

Prof. Dr. H. Heusser, 2. Chirurgische Universitätsklinik, Bürgerspital,

Basel.

Dr.G. G. Gasser

Urol. Klinik der Universität Wien.

Doz. Dr. Th. Schultheis

Barbara-Hospital

Gladbeck. 